\title{
Physical Characteristics database in healthy Japanese
}

\author{
Masatoshi Higuchi ${ }^{1}$, Akira Okada ${ }^{2}$, Seiichi Hisamoto ${ }^{1}$ \\ ${ }^{1}$ National Institute of Technology and Evaluation.(NITE) \\ ${ }^{2}$ Graduate School of Human Life Science, Osaka City University.
}

\begin{abstract} factor and statistics (e.g., mean, standard deviation, scatterplot) .

\section{Introduction}

A physical characteristics database of user was established for the healthy Japanese population (approximately 1000 subjects, 20-84 years old of age) in the National Institute of Technology and Evaluation (NITE) in 2001-2002, providing manufacturing designs. A physical characteristics database was established and released to the public in April 2002. The database consists of six parts: Basic human body measurements (BHBMS), maximum voluntary contraction (MVC), manual strength (MS), range of motion (ROM), joint passive resistance (JPR) and physical strength (PS). This report describes its construction and briefly summarizes the database.
\end{abstract}

Basic human body measurements (BHBMS), maximum voluntary contraction (MVC), manual strength (MS), range of motion (ROM), joint passive resistance (JPR), and physical strength (PS) were measured in a healthy Japanese population (approximately 1000 subjects, 20-84 years old) in 2001-2002, and the data of these have already been released on the NITE web page (http://www.tech.nite.go.jp/human/) as the" Human characteristics database". BHBMS were measured at 18 measure sites. MVC, ROM, and JPR were measured in regard to the limb joints (hand, elbow, shoulder, ankle, knee and hip). MS mean the force required to push a door open, pull a door shut, turn a door knob, and all what could the subject use either arm or hand. PS was included measurement of grip strength, sitting trunk flexion, vertical jump height, jumping reaction time, and single- leg-standing time with eyes closed. The database has search and statistical analysis function, which makes it possible to retrieve data by searching for any

Keywords: Physical characteristics database, Maximum voluntary contraction, Manual strength, Range of motion

\section{Subjects}

Measurements were made on 1000 Japanese men and women (20-84 years old) recruited and selected nationwide. The body height of the men was $163.3 \pm$ $6.82[\mathrm{~cm}]$, that of the women was $152.3 \pm 6.37[\mathrm{~cm}]$, the body weight of the men was $62.3 \pm 10.4[\mathrm{~kg}]$, and that of the women was $52.2 \pm 7.4[\mathrm{~kg}]$. The composition of the subjects are shown in Fig. 1.

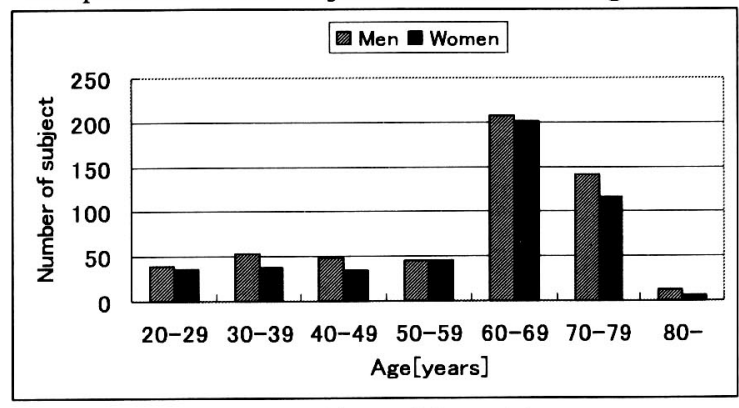

Fig. 1 Composition of the subjects

\section{Contents of database}

3.1 Basic human body measurements (BHBMS)

The BHBMS data posted in the NITE database are 18 measure positions, and they consist of four parts:
Height and weight of the body, length of segment and circumference of the segment. The height, length and circumference measurements are shown in Table 1[1] [2].

Table 1 Basic human body measurements

\begin{tabular}{|l|l|}
\hline (1) Height & (10) Thigh length \\
\hline (2) Shoulder height & (11) Length of vertex to cervicale \\
\hline (3) Elbow height & (12) Forearm circummference \\
\hline (4) weight & (13) Upper anm circummference \\
\hline (5) Hand length & (14) Thigh circummference \\
\hline (6) Forearm length & (15) Calf circummference \\
\hline (7) Upper arm length & (16) Neck circummference \\
\hline (8) Foot length & (17) Upper chest circummference \\
\hline (9) Lower leg length & (18) Abdominal extension girth \\
\hline
\end{tabular}

\subsection{Maximum voluntary contraction (MVC)}

The MVC data posted in the NITE database are isometric measurements made in the sagittal plane. The data are described maximum force and maximum torque measurements, and the torque measurements were gravity compensated by mean of the body segment parameter. MVC measurements were made of limb joints (hand, elbow, shoulder, ankle, knee, and hip). An example of measurement during knee extension is shown in Fig. 2.

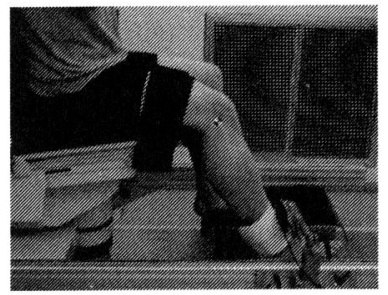

Fig.2. A measurement during knee extension (MVC)

\subsection{Manual strength (MS)}

In this part, MS was measured as the force required to push a door open, pull a door shut, turn a door knob and all what could the subject use either arm or both hands. The MS data posted in the NITE database are listed bellow, and an example of measurement of MS is shown in Figs 3, 4, 5, and 6. 
- Pushing/Pulling a door with one hand

- Pushing a button with finger

- Turning a door knob

- Gripping a cylinder
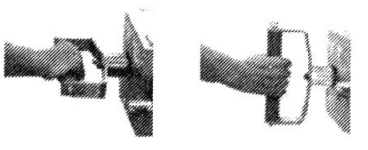

Fig. 3 Pushing/pulling a door with one hand

Fig. 4 Pushing a button with finger
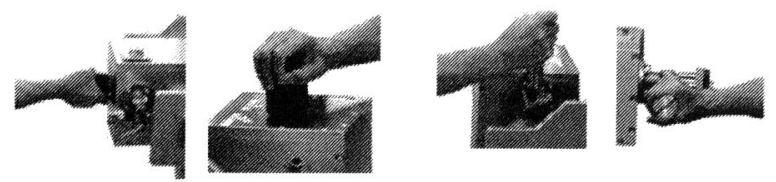

Fig. 6 Gripping a cylinder

\subsection{Range of motion (ROM)}

The ROM data posted in the NITE database are for active range of motion. The ROM data except for the shoulder joint and hip joint were measured in the sagittal plane, and the shoulder joint and hip joint data are reported for measured in the sagittal plane and horizontal plane. An example of measurement for elbow flexion in the sagittal plane is shown Fig. 7.

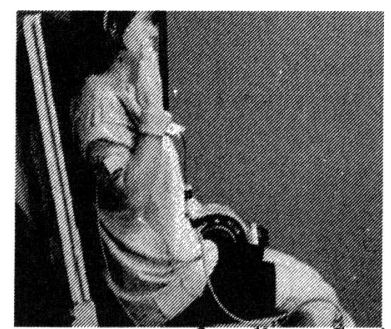

Fig. 7. A measurement for elbow flexion (ROM)

\subsection{Joint passive resistance (JPR)}

The JPR data posted in the NITE database are torque values measured during rotation of the joint without the activities muscles. The JPR data except for the shoulder joint and hip joint were measured in the sagittal plane, and the shoulder joint and hip joint data are reported for the sagittal plane and horizontal plane. An example of measurement for shoulder flexion in the sagittal plane is shown in Fig. 8.

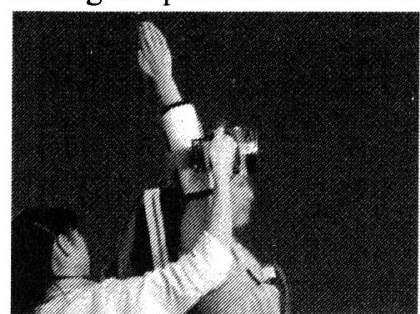

Fig. 8 A measurement for shoulder flexion (JPR)

3.6 Physical strength (PS)

The PS data posted are for:
- Grip strength

- Sitting trunk flexion

- Vertical jump height

- Jumping reaction time

- Single-leg- standing time with eyes closed

\section{Search and statistical analysis function}

The database has search and statistical analysis functions, so it is able to display raw data, statistics (e.g., means, maximum-minimum values, standard deviations) and scatterplots.

Table 2 and Fig. 9 show examples the instance of searching and statistical processing in regard to torque of elbow flexion in women.

Table 2 Statistical data

\begin{tabular}{|r|r|r|r|r|}
\hline Part & \multicolumn{1}{|c|}{ Item } & \multicolumn{1}{|c|}{ Count } & Averere & \multicolumn{1}{c|}{ SD } \\
\hline Elbow & $\begin{array}{l}\text { flexion } \\
80 \mathrm{deg} \\
{[\mathrm{Nm}]}\end{array}$ & 838 & 24.43 & 6.33 \\
\hline Max & Min & 5\%tile & 95\%tile & - \\
\hline 46.71 & 6.72 & 13.76 & 34.61 & - \\
\hline
\end{tabular}

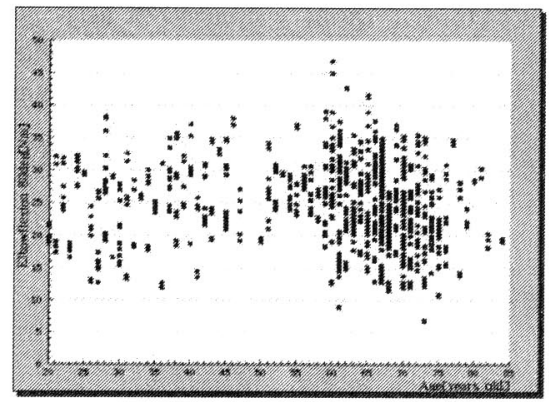

Fig. 9 Scatterplots

\section{Access to NITE web page}

Users must access to our web page (http://www.tech.nite.go.jp/human/) and register to the database to use the database. The user must also enter the purpose for which the data will be used. Once registered, the database user can make free use of the "Human characteristics database".

\section{Conclusion}

The database was released to the public in April 2002, and about one thousand people have used our database during the past four years. We plan to update and improve the database, and we hope many people will use it to effectively in future years.

\section{References}

[1] JIS Z 8500:Ergonomics-Basic human body measurements for technological design, 2002

[2] Hoshi H: Seitai no senkeisokuhou[The measurement of human body ], Terapaiya, 1989

\section{Contact}

Correspondence Address: Masatoshi Higuchi, National Institute of Technology and Evaluation No2 Annex Osaka Godo Chosha, 4-1-67,Otemae,Chuo-ku, Osaka-shi, Osaka 540-008, Japan Phone:+81-6942-1115,FAX :+81-6946-7280

e-mail :higuchi-masatoshi@nite.go.jp 